\title{
Quetiapine-Induced Severe Hypoglycemia in Patient Without Diabetes Mellitus: A Case Report
}

\author{
Suk Ju Cho ${ }^{1}$, Sang Ah Lee ${ }^{2,3}$ \\ ${ }^{1}$ Department of Anesthesiology and Pain Medicine, Jeju National University School of Medicine, Jeju, Korea \\ ${ }^{2}$ Department of Internal Medicine, Jeju National University School of Medicine, Jeju, Korea \\ ${ }^{3}$ Institute for Medical Science, Jeju National University, Jeju, Korea
}

Corresponding Author:

Sang Ah Lee, MD, PhD

https://orcid.org/0000-0002-9797-8050

Department of Internal Medicine,

Jeju National University School of

Medicine, 15 Aran 13-gil, Jeju 63241,

Korea

E-mail: peonysalee@gmail.com

Received: December 12, 2018

Revised: February 1, 2019

Accepted: February 10, 2019
Hypoglycemia is a serious event in older patients with diabetes mellitus because it increases the risk of various conditions including cardiovascular disease, and dementia, as well as the risk of falls in these individuals. Quetiapine is a widely used antipsychotic drug with diverse indications, and it may be prescribed to older patients. We report on our treatment of an older individual without diabetes mellitus who manifested severe hypoglycemia induced by quetiapine. The hypoglycemia was accompanied by endogenous hyperinsulinemia but was not related to the presence of a pancreatic tumor or antibodies, and adrenal function was normal. The hypoglycemia was so severe that only massive $20 \%$ dextrose infusion could maintain normoglycemia. After the patient stopped taking quetiapine, his blood glucose concentration started to increase slowly toward normoglycemia. We finally diagnosed this case as quetiapine-related severe hypoglycemia in a patient without diabetes mellitus, and report our findings here. (Ann Geriatr Med Res 2019;23:35-37)

Key Words: Hypoglycemia, Aged, Quetiapine fumarate

\section{INTRODUCTION}

Hypoglycemia is a serious medical condition and one of the most important medical issues in patients with diabetes, because it is primarily caused by antidiabetic medications. Hypoglycemia can contribute to or cause injuries, dementia, cardiovascular events, arrhythmias, coma, and death. ${ }^{1)}$

Quetiapine is used widely for the treatment of schizophrenic or other psychotic symptoms. Older individuals are often treated with quetiapine to relieve psychotic symptoms associated with conditions including dementia and delirium. ${ }^{2)}$ Hyperglycemia is an adverse event frequently observed in individuals without diabetes who receive quetiapine, and thus patients are monitored for these hyperglycemic episodes with 75-g oral glucose tolerance tests (OGTT). However, blood glucose concentration reductions to $47-53 \mathrm{mg} / \mathrm{dL}$ are rarely detected by a $75-\mathrm{g}$ 0GTT, although such cases have been reported. ${ }^{3-7)}$ Quetiapine is known to be able to induce mild hypoglycemia in patients without diabetes. We report on our experience treating an older individual without diabetes who developed severe hypoglycemia as a result of administration of quetiapine.

\section{CASE REPORT}

A 74-year-old man was admitted to our emergency department with abdominal pain that had begun 1 day before presentation. He complained of yellowish watery diarrhea, abdominal pain, and diaphoresis. His medical history was significant for a cerebrovascular accident (CVA), heart failure, and vascular dementia. His only prior surgeries were vertebral fixation and a cataract operation. The patient had begun to take rosuvastatin, donepezil, clopidogrel, cilostazol, and quetiapine for vascular dementia after the CVA. Along with these medications, he also took candesartan, furosemide, and carvedilol for heart failure. At the time of admission, the patient's dose of quetiapine had been increased gradually from $50 \mathrm{mg}$ to $300 \mathrm{mg}$ a day over the preceding 2 months. The dose had been changed to $300 \mathrm{mg} 1$ week prior to admission. The patient's family confirmed that he had not consumed alcohol for the past 3 years.

The patient's initial vital signs were blood pressure $96 / 64 \mathrm{mmHg}$, heart rate 68/min, and body temperature $36.6^{\circ} \mathrm{C}$. He showed only mild periumbilical tenderness on physical examination. Initial laboratory results indicated hypoglycemia ( $34 \mathrm{mg} / \mathrm{dL})$, hypokalemia $(3.2 \mathrm{mmol} / \mathrm{L})$, and elevated creatinine level $(1.92 \mathrm{mg} / \mathrm{dL})$ with a normal blood urea nitrogen $(17.1 \mathrm{mg} / \mathrm{dL})$. Other laboratory data showed 
significant changes including mildly elevated white blood cell count $\left(11,000 / \mathrm{mm}^{3}\right)$ and normal levels of aspartate aminotransferase and alanine aminotransferase, sodium, chloride, and C-reactive protein. Abdominal computed tomography (CT) showed no abnormal results related to his abdominal pain. Therefore, our clinical impression was that the patient's abdominal pain and diarrhea were caused by enteritis, and we treated him with ciprofloxacin and hydration.

The patient exhibited sustained hypoglycemia (blood glucose concentration $<40 \mathrm{mg} / \mathrm{dL}$ ) for $48 \mathrm{~h}$ after admission, with amelioration of the diarrhea (Fig. 1). Because he was consuming an insufficient amount of food (diet 2,200 kcal), the patient was administered peripheral intravenous nutritional support (glucose $225 \mathrm{~g}$, triglyceride $37.5 \mathrm{~g}$, and various amino acids). A rapid adrenocorticotropic hormone test showed a normal response with peak cortisol level of $22.5 \mu \mathrm{g} / \mathrm{dL}$, and we excluded adrenal insufficiency as a cause of hypoglycemia. The other results were glucose $42 \mathrm{mg} / \mathrm{dL}$, serum C-peptide $19.17 \mathrm{ng} / \mathrm{mL}$, insulin 66.92 $\mu \mathrm{U} / \mathrm{mL}$, and hemoglobin A1c level 5.7\%. These results suggested that the cause of hypoglycemia was endogenous hyperinsulinemia. However, CT scans showed no masses in the pancreas or other organs.

The patient in this report usually runs and walks for $2 \mathrm{~h}$ after rising each morning prior to eating anything and had never previously experienced hypoglycemic symptoms. The mentioned blood test and CT scan results indicated that his hypoglycemia was not caused by insulinoma or nesidioblastosis, the main causes of endogenous hyperinsulinemia. We also excluded autoimmune hypoglycemia because the test results were negative for anti-insulin antibody and anti-insulin receptor antibody. This patient's hypoglycemia was very severe, and his blood glucose level remained $<50 \mathrm{mg} / \mathrm{dL}$ even with administration of $10 \%$ dextrose at $160 \mathrm{~mL} / \mathrm{h}$. We therefore decided to infuse a higher concentration of glucose via a peripherally inserted central catheter. We also treated the patient with gluco-

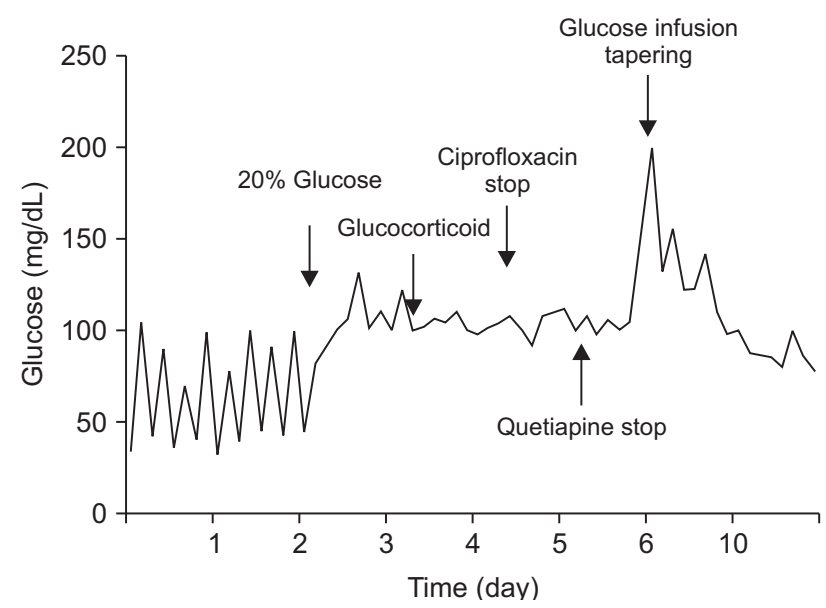

Fig. 1. Change in blood sugar levels according to clinical course. corticoid $300 \mathrm{mg}$ per day via continuous infusion, but this had no effect on his hypoglycemia. The patient's blood glucose level was maintained at $>100 \mathrm{mg} / \mathrm{dL}$ after infusion of 20\% dextrose $100 \mathrm{~mL} / \mathrm{h}$.

Finally, we attempted to exclude drugs as a cause of hypoglycemia, and decided to sequentially eliminate drugs with a high potential for hypoglycemia. We first stopped ciprofloxacin and then quetiapine $24 \mathrm{~h}$ later (although we noted that quetiapine is rarely a cause of hypoglycemia). Following termination of quetiapine, the patient's blood glucose level started to increase, and we were able to decrease the rate and concentration of dextrose fluid and eventually stop this infusion. Laboratory data showed levels of C-peptide $1.1 \mathrm{ng} / \mathrm{mL}$, insulin $6.9 \mu \mathrm{U} / \mathrm{mL}$, and blood glucose $84 \mathrm{mg} / \mathrm{dL}$ on the day prior to the patient's discharge, and he was discharged without any complications. The patient has experienced no symptoms related to hypoglycemia in the past 6 months.

\section{DISCUSSION}

The population is aging worldwide, and the prevalence rates of several diseases, including cerebrovascular disease, dementia, cardiovascular disease, Parkinson's disease, and diabetes mellitus, are increasing in the older population. Korea and Japan have the highest percentages of older individuals worldwide. ${ }^{8)}$ Quetiapine is widely used to relieve psychotic symptoms in older patients with various conditions. Quetiapine can induce hyperglycemia as a minor adverse event, and mild hypoglycemia is a rare adverse event that is usually without symptoms. Previous reports show that quetiapine can induce mild hypoglycemia and that most cases are detected accidentally when the patient is examined for hyperglycemia. ${ }^{3-7)}$

In the present case, quetiapine-induced hypoglycemia was more severe than that described in previous reports, and was sufficiently severe to require a massive $20 \%$ dextrose fluid infusion. Quetiapine, a second generation antipsychotic drug, has an high affinity for and an antagonistic effect on the histamine $\mathrm{H} 1$, adrenergic $\alpha 1$, muscarinic $\mathrm{M} 1,5-\mathrm{HT}_{2 \mathrm{~A}}$, 5- $\mathrm{HT}_{7}$, dopamine $\mathrm{D}_{2}$, and $\mathrm{D}_{3}$ receptors. ${ }^{9)}$ Second generation antipsychotic drugs exert their effects through these various receptors. By interacting with these receptors, quetiapine may increase the amount of basal insulin secreted by pancreatic $\beta$-cells and thereby cause hypoglycemia. ${ }^{10)}$ Insulin is secreted by pancreatic $\beta$-cells independent of other types of hormone-secreting cells, which together with $\beta$-cells form the islets of Langerhans. ${ }^{10)}$ Quetiapine is an antagonist of muscarinic receptors and may influence the action of insulin through its effects on these receptors; this is another possible mechanism to explain quetiapine-related hypoglycemia. ${ }^{9)}$ However, the mechanism underlying quetiapine-induced hypoglycemia remains unclear, and further studies are required.

The present case demonstrates that quetiapine can be a cause of severe hypoglycemia in older individuals, even 
those without diabetes. Since quetiapine is used for the relief of psychotic symptoms, it is crucial to understand the potential adverse effects related to the use of this medication, and to observe any symptoms that may appear. One case report described blonanserin as another option for improving hypoglycemia related to quetiapine use. ${ }^{5)}$ We suggest that clinicians should carefully observe older patients receiving quetiapine to identify any hypoglycemic symptoms that may appear. If the etiology of hypoglycemia is not clear in patients using quetiapine, it is important to consider quetiapine as a possible cause.

\section{CONFLICTS OF INTEREST DISCLOSURES}

The researchers claim no conflicts of interest.

\section{REFERENCES}

1. Yamada T, Shojima N, Noma H, Yamauchi T, Kadowaki T. Glycemic control, mortality, and hypoglycemia in critically ill patients: a systematic review and network meta-analysis of randomized controlled trials. Intensive Care Med 2017;43:1-15.

2. Kim MY, Etherton-Beer C, Kim CB, Yoon JL, Ga H, Kim HC, et al. Development of a consensus list of potentially inappropriate medications for Korean older adults. Ann Geriatr Med Res 2018;22:121-9.

3. Fujita T, Mizoguchi Y, Kunitake Y, Tateishi H, Inaba T, Kato TA, et al. Second-generation antipsychotic-induced hypoglycemia. Prim
Care Companion CNS Disord 2018;20:17br02186.

4. Mancano MA. Pancreatitis-associated with riluzole; linezolidinduced hypoglycemia; sorafenib-induced acute generalized exanthematous pustulosis; creatine supplementation-induced thrombotic events; acute pancreatitis associated with quetiapine; hypomagnesemia and seizure associated with rabeprazole. Hosp Pharm 2014;49:1004-8.

5. Suzuki Y, Tsuneyama N, Sugai T, Fukui N, Watanabe J, Ono S, et al. Improvement in quetiapine-induced hypoglycemia following a switch to blonanserin. Psychiatry Clin Neurosci 2012;66:370-1.

6. Suzuki Y, Watanabe J, Fukui N, Ozdemir V, Someya T. Hypoglycaemia induced by second generation antipsychotic agents in schizophrenic non-diabetic patients. BMJ 2009;338:a1792.

7. Watanabe A, Fukui N, Suzuki Y, Motegi T, Igeta H, Tsuneyama $\mathrm{N}$, et al. Improvement of dumping syndrome and oversecretion of glucose-dependent insulinotropic polypeptide following a switch from olanzapine to quetiapine in a patient with schizophrenia. Gen Hosp Psychiatry 2015;37:372.e5-6.

8. Suzuki T. Health status of older adults living in the community in Japan: recent changes and significance in the super-aged society. Geriatr Gerontol Int 2018;18:667-77.

9. Bymaster FP, Felder CC, Tzavara E, Nomikos GG, Calligaro DO, Mckinzie DL. Muscarinic mechanisms of antipsychotic atypicality. Prog Neuropsychopharmacol Biol Psychiatry 2003;27:1125-43.

10. Melkersson K. Clozapine and olanzapine, but not conventional antipsychotics, increase insulin release in vitro. Eur Neuropsychopharmacol 2004;14:115-9. 